\title{
Cutaneous manifestations of infections: an update for general physicians
}

\author{
Author: Rachael Morris-Jones ${ }^{A}$
}

Infection rashes can present in a number of different ways, some giving a reactive pattern in the skin to infection elsewhere in the body, while others reflect infection within the affected skin itself. Recognising the possible systemic symptoms, characteristic distribution and morphology of rashes can lead to a high degree of accuracy in making a clinical diagnosis in the clinic room/at the bedside that can be confirmed with a few simple laboratory tests.

\section{Cutaneous manifestations of SARS-CoV-2 (COVID-19)}

There are five main skin manifestations of COVID-19 infection in adults, the prevalence of skin manifestations has been estimated to be between $0.4-20 \%$. The three main skin presentations are similar to those you might see with other systemic viral infections, maculopapular rash (similar to what you might see in measles), vesicles (chicken pox-like blisters) and urticaria (hives). These rashes occur at the same time as viral symptoms and are usually associated with mild to moderate symptoms of COVID-19. 'COVID-toes' are a late manifestation of usually very mild SARSCoV-2 infection (patients may otherwise be asymptomatic) and present with chilblain-like lesions on the toes, fingers and elbows (Fig 1). There is dusky purplish erythema and blistering which may be asymptomatic and is thought to be due to microthrombi caused by the virus within the endothelial cells. The fifth cutaneous manifestation is livedoid/retiform purpura which is associated with more severe COVID-19 disease (thrombotic vasculopathy) and usually occurs at the same time or later in the course of the COVID-19 infective illness. There is no specific skin directed treatment needed for any of these cutaneous manifestations.

\section{Varicella-zoster infections (shingles)}

Varicella-zoster is a herpes virus that remains latent in the sensory root ganglion after primary chicken pox infection, which can then reactivate later in a dermatomal distribution as shingles. Before the appearance of the shingles skin lesions, there may be a prodrome of malaise, headache and tingling within the skin or even pain prior to the appearance of the small red papules, blisters, 'punched-out' erosions and crusting (Fig 2). Haemorrhagic

Author: Adermatology consultant, St John's Institute of Dermatology, London, UK changes and pustules may also be seen, especially in elderly patients. Lesions are infectious for 7-10 days until they crust over. The most commonly affected dermatomes are thoracic and lumbar. Involvement of the ophthalmic branch of the trigeminal cranial nerve (herpes zoster ophthalmicus) can lead to ocular damage and there is a $50 \%$ increased risk of having a cerebrovascular accident (CVA; stroke) especially within the first 2 weeks but the increased risk seems to last at least 3-6 months. Patients should be advised to seek immediate medical assessment if they experience transient ischaemic attack/stroke symptoms (can be thrombotic or haemorrhagic CVA). It is uncertain if treating with antiviral therapy reduces the risk of stroke, however, it would be prudent to do so as this can reduce viral shedding, reduce the duration and severity of the neuritis, hasten healing and may lessen the risk of post-herpetic neuralgia (reduced by $46 \%$ in studies). ${ }^{2}$ It is best to start antiviral therapy within 72 hours of the onset to gain the most benefit. Treat for 7 days with oral

\section{Key points}

If a patient presents with symptoms of a viral illness and a skin rash, think of SARS-CoV-2 (COVID-19). COVID toes can present mimicking chilblains in patients who are otherwise asymptomatic.

Herpes zoster ophthalmicus increases the risk of a cerebrovascular accident within 2 weeks by $50 \%$.

Recombinant glycoprotein $\mathrm{E}$ (Shingrix) is more effective at preventing shingles and reducing the frequency of postherpetic neuralgia in patients over the age of 60 years than the live vaccine.

Atypical mycobacterial skin infections can arise in the UK and present with red/purple nodules, tissue culture and PCR are helpful to guide choice of antibiotics.

Scabies is common in overcrowding and in institutions. Look out for and treat secondary bacterial infection to prevent acute and chronic complications of Streptococcus/ Staphylococcus.

KEYWORDS: cutaneous manifestations, COVID-19, scabies, shingles, varicella-zoster virus

DOI: $10.7861 /$ clinmed.2021-0262 


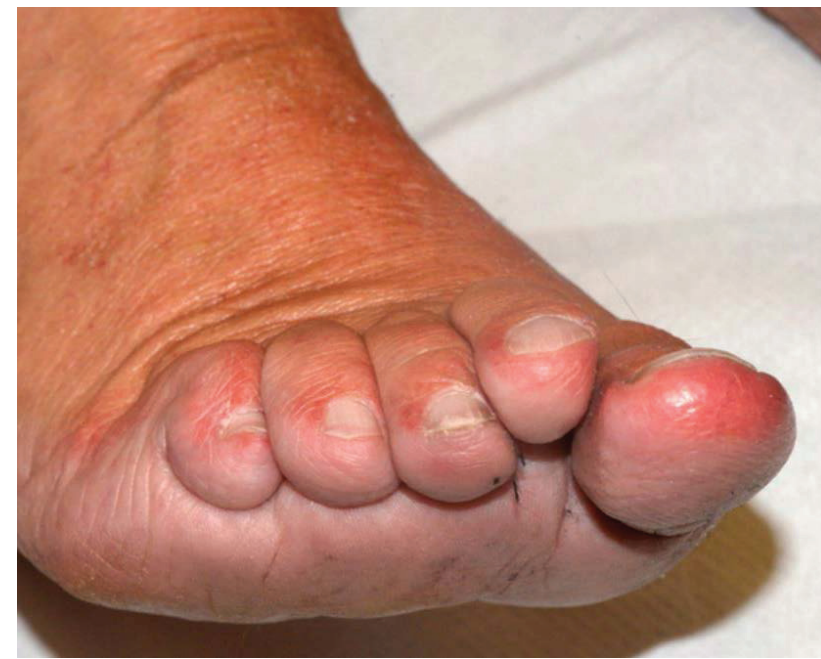

Fig 1. COVID toes.

aciclovir $800 \mathrm{mg} 5$ times a day, valaciclovir ( $3-5$ fold increase bioavailability than aciclovir) $1,000 \mathrm{mg}$ three times or famciclovir (prodrug of penciclovir) $500 \mathrm{mg}$ three times a day. Secondary bacterial infection of the skin lesions is common, therefore an antiseptic wash introduced early can help to prevent this, or treat with anti-staphylococcal oral antibiotics. Paraffin (50:50 of white soft paraffin to liquid paraffin) should be applied to the affected skin to keep it moist and protected from secondary colonisation. Disseminated zoster (multiple skin lesions in a generalised distribution distant from the main dermatome affected) may be a sign of underlying immunodeficiency (haematological malignancies and transplant recipients). Antiviral treatment should be given to all immunocompromised patients with zoster even if they present after 72 hours, usually with intravenous aciclovir.

In the UK, patients over the age of 70 years can be given zoster immunisation to try to boost their cell-mediated immunity against zoster, 50 patients need to be vaccinated to prevent one person

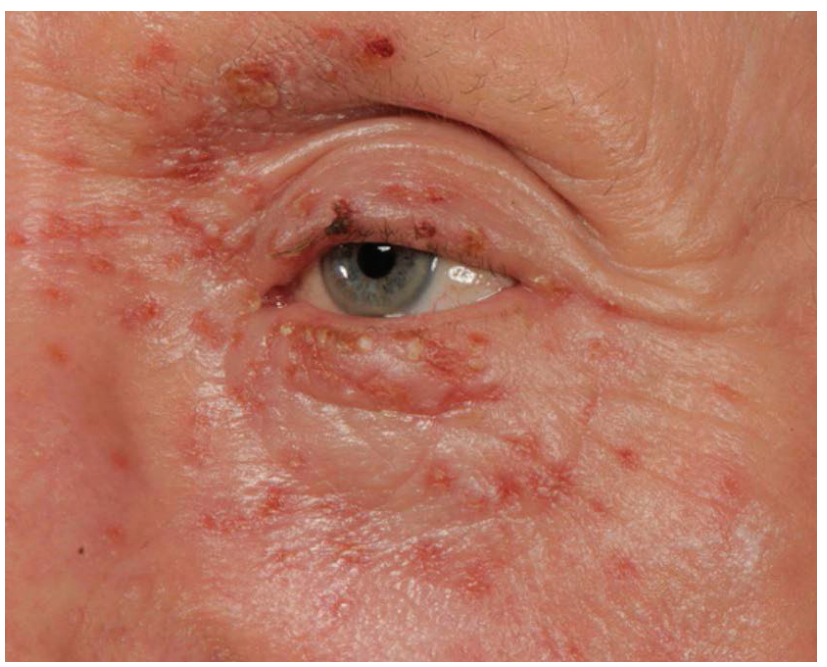

Fig 2. Herpes zoster.

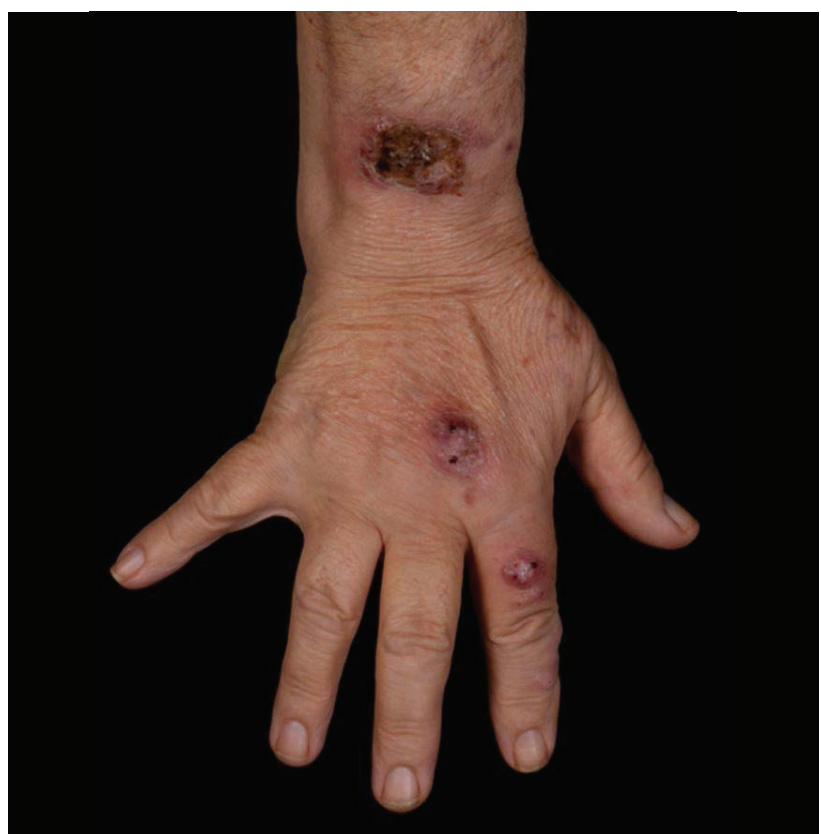

Fig 3. Sporotrichoid spread.

getting shingles. Recombinant glycoprotein E (Shingrix) or live attenuated (Zostavax) can be given. There is some evidence that Shingrix is more effective at preventing shingles and reducing the frequency of post-herpetic neuralgia in patients over the age of 60 years than the live vaccine. ${ }^{3}$

\section{Cutaneous atypical mycobacterial infection}

Atypical mycobacterial skin infections can arise in the UK usually from direct skin contact with the microorganisms in the environment (water, soil, plant material and tattoos) or from infected fish (tropical fish tank/fish spa or open water fish). Lesions are often solitary but may be multiple, depending on the exposure and the species of mycobacteria causing infection. Immunocompromised patients may get disseminated disease in the skin. Lesions tend to start as a small erythematous/purplish papule or nodule that grows in skin over weeks to months and may discharge pus and may ulcerate. Lesions may be tender or painful

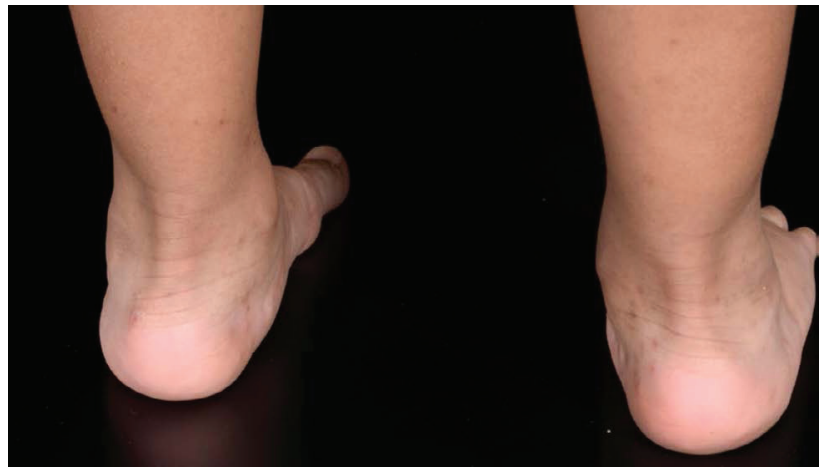

Fig 4. Scabies. 
but can also be asymptomatic. Common species causing disease in the UK include Mycobacterium marinum (lesions usually on the dominant hand and may spread up the arm, so-called sporotrichoid spread (Fig 3)). Rapidly growing atypical mycobacteria include $M$ abscessus, $M$ chelonae and $M$ fortuitum. Diagnosis is usually based on skin biopsies taken from lesional skin and processed for histology and culture/sensitivity testing. Culture and PCR is needed for species identification, which can, alongside sensitivity testing, help guide anti-mycobacterial therapy. Treatment is usually required for between 4-6 months, or 6 weeks past clinical cure. Small isolated nodules at an amenable site could be excised if prolonged antimicrobial treatment is not effective/possible.

\section{Scabies and secondary bacterial infection}

The World Health Organization has recently listed scabies as a neglected tropical disease. Prevalence is highly variable throughout the world from $0.2-71 \%$ of the population affected. ${ }^{4}$ Outbreaks can occur in overcrowded housing, long-term care facilities and prisons. Spread is usually through direct close contact (at least 20 minutes skin-to-skin contact) and also less commonly through contact with fomites (mites can survive off their host for up to 36 hours). In classic scabies, there are usually 10-15 mites infesting an individual's skin, in crusted scabies there can be millions, leading to an increased risk of spread through fomites. Symptoms of intense, mainly nocturnal, itching start 3-6 weeks after acquiring the infestation. The clue to the diagnosis is usually in the history with multiple family members simultaneously affected by an itchy skin condition. Clinically, appearances are highly variable with papules, excoriations and possibly burrows (Fig 4). Classically, hands/wrists and genital areas are affected in adults, in young infants, the axillae and soles of the feet may be involved. Crusted scabies presents with fine scale, particularly over the extremities. Secondary Staphylococcus and Streptococcus are important secondary complications of scabies, which is often overlooked. This may lead to impetigo, furunculosis, sepsis and even poststreptococcal glomerulonephritis. Treat the patient and their close personal contacts simultaneously with either topical permethrin or oral ivermectin. Pernethrin 5\% cream should be applied to all skin sites from the neck downwards in adults (include the scalp in babies) and left on for 12 hours and repeated after 7 days (as the treatment is not ovicidal). Oral ivermectin $200 \mu \mathrm{g} /$ $\mathrm{kg}$ as a single dose or repeated at 7 days (not recommended in pregnancy and children who weigh $<15 \mathrm{~kg}$ ) is especially helpful in managing institutional outbreaks. Treat crusted scabies with $200 \mu \mathrm{g} / \mathrm{kg}$ for 3-7 consecutive days depending on the severity. Treat secondary bacterial infection with systemic antibiotics and topical antiseptic washes to prevent long-term sequela. Itching usually takes 6 weeks to settle post-successful treatment of scabies, so patients should be prescribed a topical steroid and an emollient to help supress the ongoing skin reaction. Moxidectin currently used to treat onchocerciasis ( $8 \mathrm{mg}$ single dose) may supersede ivermectin if phase III trials show single dosing has a superior efficacy. This would be very attractive for use in mass eradication programmes globally.

\section{References}

1 Conforti C, Dianzani C, Agozzino M et al. Cutaneous manifestations in confirmed Covid-19 patients: A Systemic Review. Biology 2020;9:449.

2 Jackson JL, Gibbons R, Meyer $\mathrm{G}$ et al. The effect of treating herpes zoster with oral acyclovir in preventing postherpetic neuralgia. A meta-analysis. Arch Intern Med 1997;157:909.

3 McGirr A, Widenmaier R, Curran D et al. The comparative efficacy and safety of herpes zoster vaccines: A network meta-analysis. Vaccine 2019;37:2896.

4 Romani L, Steer AC, Whitfield MJ et al. Prevalence of scabies and impetigo worldwide: a systematic review. Lancet Infect Dis 2015;15:960-7.

Address for correspondence: Dr Rachael Morris-Jones, St John's Institute of Dermatology, King's College London, 9th Floor Tower Wing, Guy's Hospital, London SE1 9RT, UK.

Email: rachael.morris-jones1@gstt.nhs.uk 